\title{
For Whom the Games Toll: A Qualitative and Intergenerational Evaluation of What is Serious in Games for Older Adults
}

\author{
Najmeh Khalili-Mahani, 2,3,4,7 $\cdot$ Bob De Schutter ${ }^{5} \cdot$ Mahsa Mirgholami $^{1,4}$. \\ Eileen Mary Holowka ${ }^{3,6} \cdot$ Rebecca Goodine $^{3} \cdot$ Scott DeJong ${ }^{3,6}$. \\ Roseleen McGaw ${ }^{8} \cdot$ Sue Meyer $^{8} \cdot$ Kim Sawchuk $^{2,3,6}$
}

Published online: 11 March 2020

(C) The Author(s) 2020

\begin{abstract}
The aim of this study was to engage older adults in discussions about digital serious games. Using a qualitative exploratory approach, we report observations from more than $100 \mathrm{~h}$ of conversations with individuals in the age range 65-90, in a study entitled "Finding better games for older adults" (June 2017-December 2019). Phase 1 (19 older participants, 3 young research students) involved conversations around a quantitative study of cognitive benefits of digital playing (minimum $6 \mathrm{~h} /$ person). Phases 2 and 3 involved a focus group in the form of a community class (10 weeks, $2 \mathrm{~h}$ per meeting), involving introduction to digital game genres, playing, and discussing motivations and obstacles for current and future play. Cognitive stimulation, emotional distraction and physical therapy were initially stated as the motives for game play. However, with growing familiarity and voluntary exchanges of personal stories between older and younger participants, the cultural significance of the medium of game (especially with story-telling and VR technology) became more important to older adults. More than mechanical inaccessibility, lack of access to the cultural discourse about games presents barriers for older adults. To create a safe, comfortable and accessible space for intergenerational learning and play is of primary importance both for users and designers, should serious games be considered for the future of digital care.
\end{abstract}

Keywords Serious games $\cdot$ Intergenerational games $\cdot$ Accessibility $\cdot$ Games for health · Game types · Focus group

Najmeh Khalili-Mahani

najmeh.khalili-mahani@concordia.ca

Extended author information available on the last page of the article 


\section{Introduction}

According to a $2015 \mathrm{UN}$ report, the world's aging population (60 years + ) will reach 1.4 billion people in 2030-outnumbering children (less than 9) by 0.1 billion-accounting for $25 \%$ of the total world population. This inter-generational imbalance demands innovative approaches to assistive care technologies. Among these approaches, gamification is a promising strategy for digital healthcare (de Vette et al. 2015).

Games, as possible activities to benefit one's health, have been studied in older adults since the 1980s (Weisman 1983; McGuire 2010; Riddick et al. 1987; de la Hera et al. 2017; Marston et al. 2016). Gamification studies focus on the health benefits of mental and social engagement with games (Loos and Kaufman 2018; Marston and Smith 2012), and their efficacy in enhancing physical (Marston et al. 2015b; Miller et al. 2014) and cognitive fitness of older adults (Zhang and Kaufman 2016a; Bleakley et al. 2015; Hall et al. 2012; Lee et al. 2012; Basak et al. 2008).

Gamification (Deterding et al. 2011) comes with an added potential advantage, especially to healthcare research (Marston and Hall 2016). Compared to standard neuropsychological or kinesiological tests, games are designed to be interactive. Thus, the scope of cognitive or physical data collection is increased by allowing researchers to track individual choices and adaptive strategies. They are also designed to be persuasive, by providing pleasure and a purpose in one's daily activities. As such, they offer a more ecological tool for behavioral or movement evaluations, when needed. For example, Vallejo et al. designed a serious game, involving six daily living tasks that tested how participants who navigated a shop, completed a shopping list, and performed on a cooking task. They showed that the game data was effective in detecting behavioral differences between those with Alzheimer's disease and healthy controls (Vallejo et al. 2017). Ouellet et al. (2018) have validated a virtual reality shop environment, as a tool for testing episodic memory in older adults. More recently, Coughlan et al. (2019) used the game app Sea Hero Quest to investigate the interindividual and demographic factors that affected navigational strategies of over 27,000 players, and found a superior diagnostic utility of this game, compared to standard memory tests, for pre-clinical detection of "at-genetic-risk" of Alzheimer's disease individuals.

From a neuroscientific and biomedical engineering perspective, we were interested in exploring the applicability of including games into digital healthcare and assistive care strategies for seniors. The aim of this research was to assess the extent of familiarity of older adults with the concept of serious games, and the appeals or barriers for their adoption into their lifestyle.

\subsection{Background}

To play for health benefits seems to be a strong motivation for interest in digital games among older adults (Whitcomb 1990; Allaire et al. 2013; Whitlock et al. 
2011; Boot et al. 2016; Marston et al. 2015a). The experiences of engaging with a meaningful, aesthetic and enjoyable challenge are more important (De Schutter and Vanden Abeele 2015; Marston et al. 2015b). Surveying Western Canadian older adults, Kauffman and colleagues reported that more than $50 \%$ (out of 875 of $55+$ years of age) played digital games, especially puzzle $(\sim 47 \%)$ or card/board games ( 29\%). Interestingly, the majority $(\sim 70 \%)$ believed that playing games were related to improved cognitive functions (e.g. memory and attention).

Comparatively, fewer perceived social or emotional benefits-at most, $42 \%$ believed that games helped them gain self-confidence, and $34 \%$ perceived them as beneficial in dealing with loneliness. These results are consistent with a study by Cota et al. (2015) who experimented with a game designed for seniors (Viajando pelo Mundo or "Traveling around the World"), and found that, while they preferred casual games, they frequently referred to the importance of playing in relation to mental exercise, brain, thinking and mind.

These observations have motivated several game classification studies to identify the best types or genres of games for older adults (De Schutter 2011; Kaufman et al. 2016; Chesham et al. 2017). Several game designers have provided design frameworks that aim to accommodate seniors' cognitive and executive needs (Gerling et al. 2011; Bouchard et al. 2012), needs for self-efficacy and connection (De Schutter 2017), and needs for mental challenge (Cota et al. 2015).

Gamification studies often focus on features of gaming hardware, software or the clinical domains of applicability (Marston and Smith 2012). Accessibility initiatives such as the International Game Developer Association Special Interest Group (https://igda-gasig.org/) have made great strides over the past decade generating awareness of accessibility needs for older adults among commercial game developers. However, games designed for older players are not commercially available yet. The interfaces and game mechanics of commercial games are still developed by and for younger markets, or a small group of proficient older players, and have been shown to impede access for older adults who are unfamiliar with computer games (Ijsselsteijn et al. 2007; Gerling et al. 2015), unless such activities are continuously guided (Hausknecht et al. 2015).

On the other hand, the human factors that determine inter-individual variation in values, culture and aesthetics appreciations are important to consider in scientific explorations of games for health (Marston et al. 2015a; De Schutter and Vanden Abeele 2015; Vanden Abeele and De Schutter 2010). In studying the interactions between two complex psychobiological constructs (aging and game as a cultural medium), theoretical restrictions, and model-driven methodologies could be hindering. Longitudinal trials that focus on effect or uptake of one type of game, or crosssectional surveys about the previous experience with game play are sophisticated in scientific design, but do not ask: "which cultural gaps are there to bridge before embarking on gamified digital healthcare?" 


\subsection{Study Objectives}

The aim of this study was to gain an understanding of how older adults relate to the notion of digital serious games as innovative technologies for enhancing their health and wellbeing. We took a qualitative approach to the questions, and adopted the approach from Jaycox and Hicks' (1976) study, in which young students and older adults were brought together to devise a program to promote computer training and usage in the elderly (CUE) (Jaycox and Hicks 1976).

Jaycox and Hicks postulated that by involving high-school students in teaching computer use to older adults, they would help bridge an intergenerational gap, in particular by sensitizing the young students to the development of 'humanized' computer programs. In their study, they found that older adults identified applications that were more pertinent to them than the intended use of technology, offered by training. Namely, for older participants in that study, playing games on computers was the preferred use case for information technologies as it enabled older generations to share experience and knowledge with younger generations, by affording hypertexts and simulated environments.

Our initiative "Finding better games for older adults", was announced in June 2017, and has been ongoing, with primary focus on participatory activities aimed at introducing seniors to different types of existing commercial games. We hypothesized that by providing an opportunity for an encounter between seniors and game research and engineering students, we would have a better understanding of the affordances of serious games for health and wellness applications that target older adults.

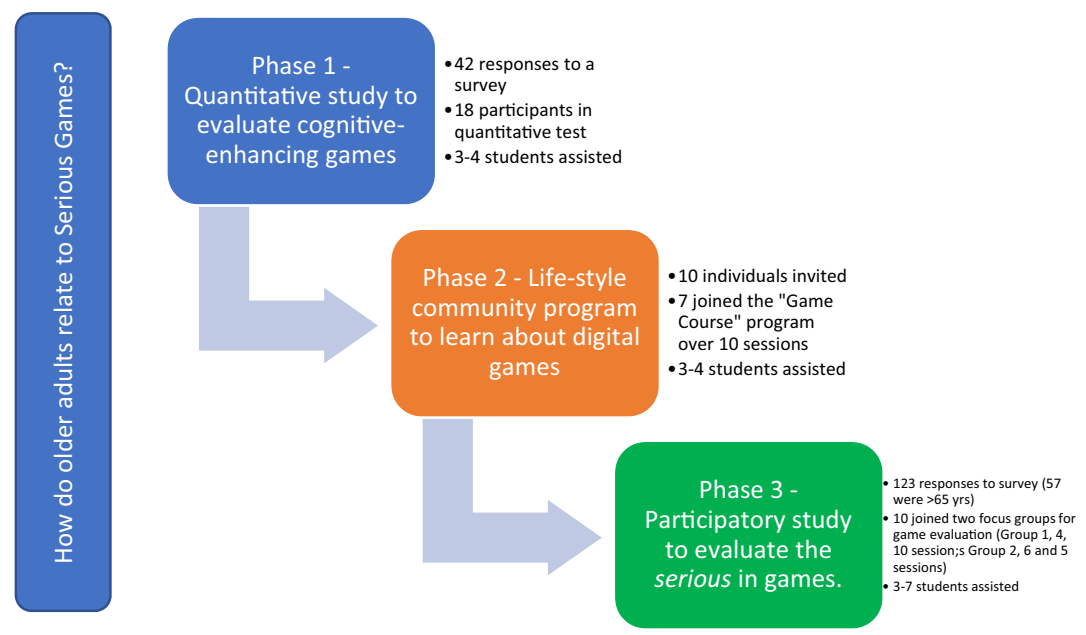

Fig. 1 Diagram of the three phases of the study 


\section{Methods}

\subsection{Experimental Procedure}

Figure 1 summarizes the three phases of our research process. The list of games is presented in Table 2.

In Phase 1, individuals between ages of 65-80 were invited to participate in the study titled "Finding Better Games for Older Adults: an objective assessment of interactions between appraisal, arousal and cognitive benefits of electronic playing".

Phase 2 grew from the findings of Phase 1, when we recognized that, besides health benefits, seniors were intrigued by the idea of digital games as cultural products. Hence, we organized a community program under the title Game Clinic Course, to introduce digital games to seniors. In this phase, we invited graduate students in game design and engineering to join a 10-weeks game-teaching activity, involving talking about different genres of games, tutorials about game making, followed by playing and discussing different games.

In Phase 3, the activities of Phase 2 were presented as a participatory research program inviting seniors to join a conversation on "What is serious about games for seniors?" The class was offered to two groups.

\subsection{Recruitment}

In all three phases, participant recruitment was conducted via a mailing list and newsletter of PERFORM Centre, reaching individuals interested in preventative health and wellness research studies in Montreal, Canada. Participation involved completing an anonymous online screening survey about game attitude. Those who wished to further continue could then contact the research team by telephone or email to schedule the upcoming activities. In order to minimize the risks of discomfort by implied judgement about self-efficacy, in Phase 2 and 3 of the study, we intentionally avoided asking questions about age and profession in the focus group unless the participants volunteered information, however all participants did confirm that they were older than the retirement age (65, in Canada).

\subsection{Data Collection}

Data collection was conducted after receiving approval from the ethics review board at Concordia University. In all three phases, the principal investigator (PI) was present in all sessions and at least two students (always the same) and the PI herself were involved in taking notes based on semi-structured conversations with specific emphasis on "What is your history of game play?", "What do you find promising in application of digital games for seniors?", and "What do you find problematic about adding digital games to your lifestyle?". 
Additionally, in Phase 2 and 3 we invited all participants (students and seniors) to write comments about their experiences in the class and reflect on how the class activities informed the respective participant(s) about their conceptualization of games. At all times, the research team maintained a collegial and conversational atmosphere, and invited candid and critical comments by all participants (students and older adults). For this reason, we did not audio- or video-record the activities or conversations, but took notes, and encouraged participants to share their thoughts in writing as well.

\subsection{Data Extraction}

We examined all the conversations case by case, and focused on statements that addressed the following questions:

- Are there any aspects of digital gaming for health that are particularly promising or stressful to seniors?

- What are the sources of intergenerational disconnect? and,

- Which aspects of digital gaming are commonly appealing to both generations?

In Phase 1, we split the statements made by participants to positive and negative, and identified areas of risk, gap, difficulty, as well as benefit, and advise for improvements. In Phases 2 and 3, we asked for written statements, and provide direct quotations that address questions above, in addition to questions about personal relations to play in childhood and older age.

\section{Results}

\subsection{Phase 1: What are the Stressful and Rewarding Aspects of Games for Health?}

The first study in Phase 1 was conceived in the theoretical framework of stress research (AGPHA) (Khalili-Mahani and De Schutter 2019), and we expected that motivation to engage with unfamiliar challenges would depend on the degree to which players counterbalanced the initial stress of learning a new (supposedly brainenhancing) game against its potential reward (expected by the participant).

On the post-game questionnaire, almost everyone stated that the experience of different sessions (in Phase 1) was enjoyable and interesting (Khalili-Mahani et al. 2020). However, some games were more stressful to some than others. A recurring source of frustration was performance, especially in individuals who cared about cognitive benefits, and valued scoring high. Another source of stress was lack of flow. If a game's visuals were too intense (crowded, confusing, distracting), or if the game dynamics were not rewarding or too challenging to learn, that would be frustrating. Nevertheless, despite acknowledging the potential benefits of the games for cognitive, emotional or physical stimulation, almost everyone preferred their nondigital hobbies and enrichment programs (craft, music, exercise, social play). 


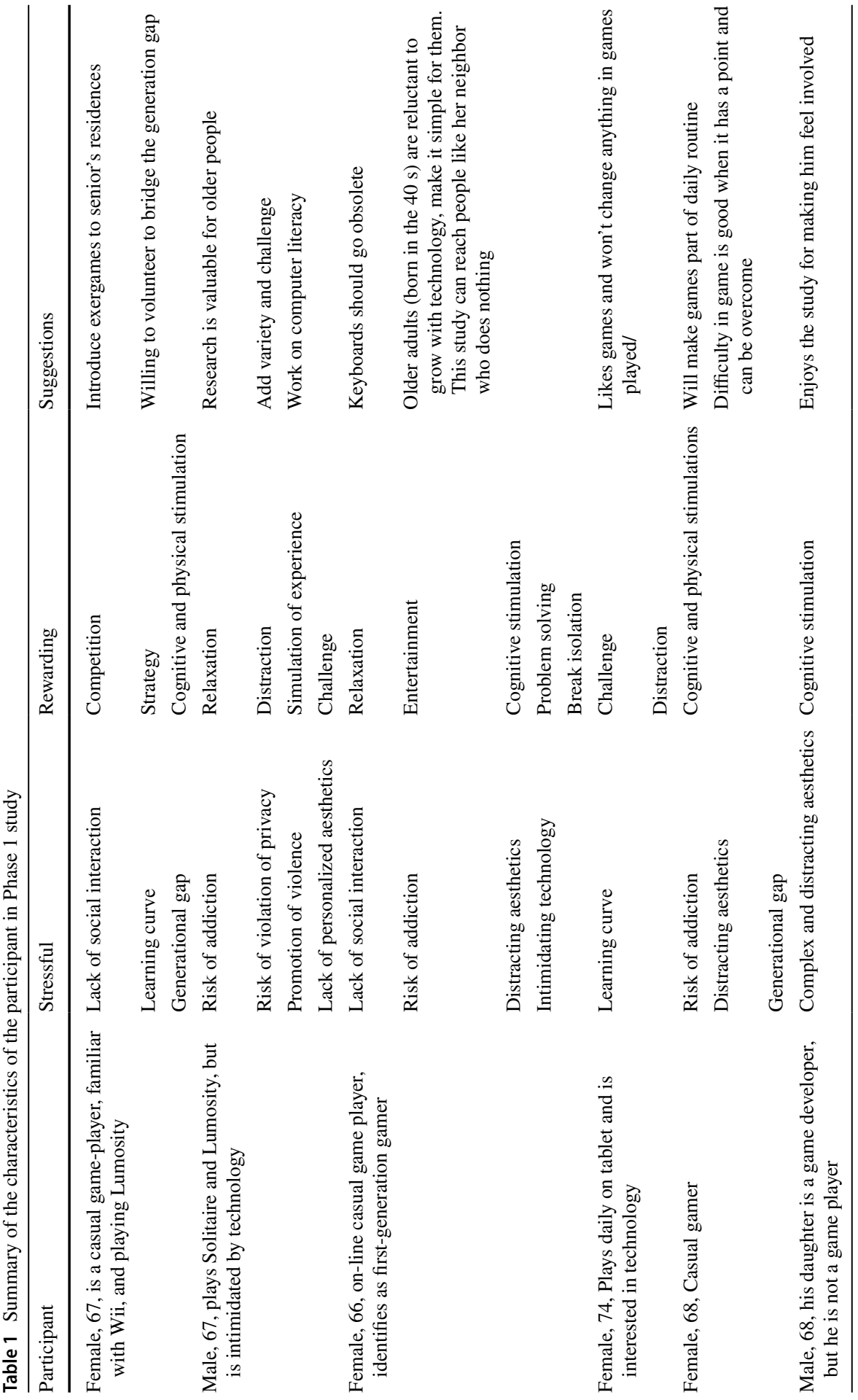




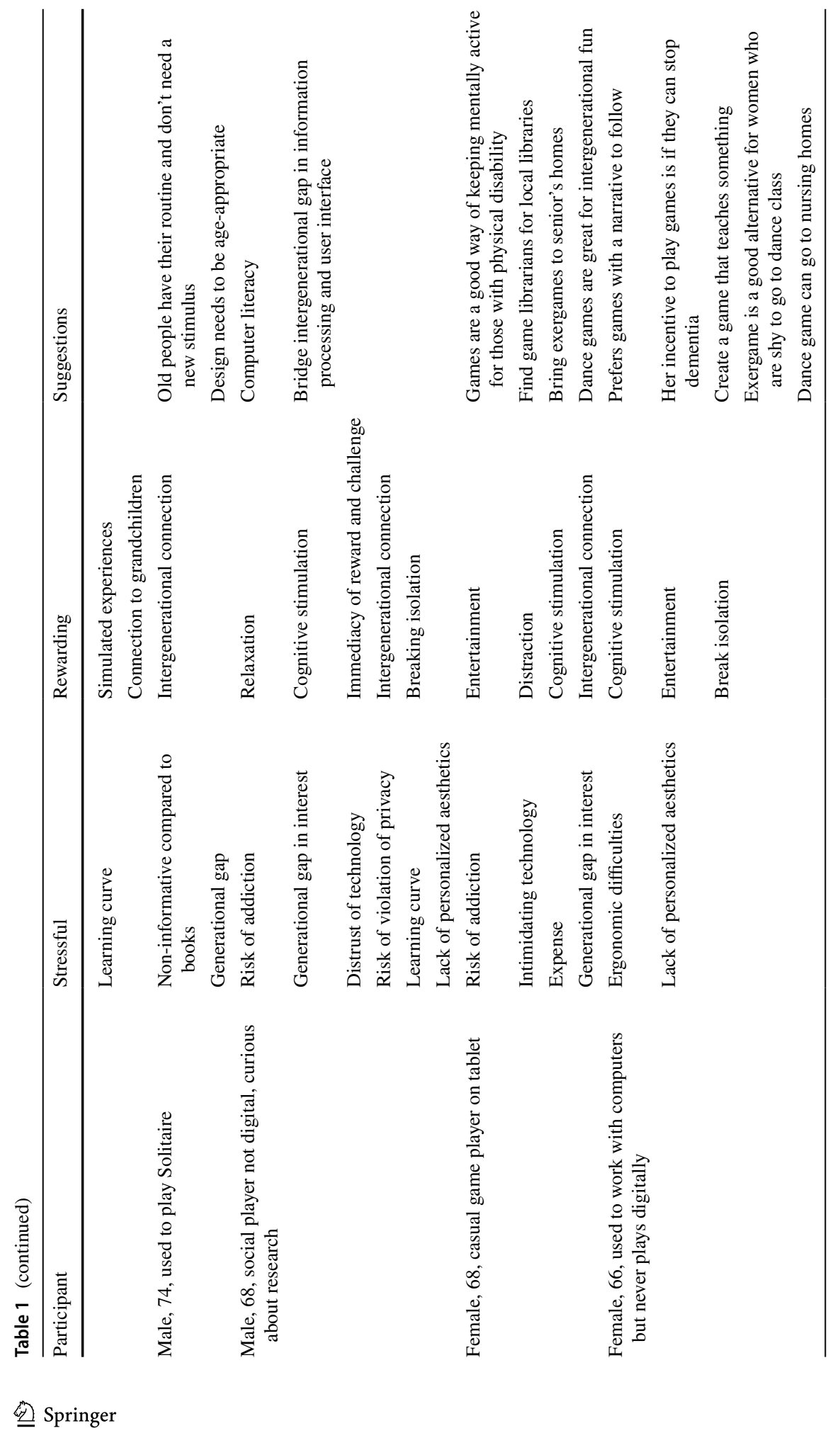




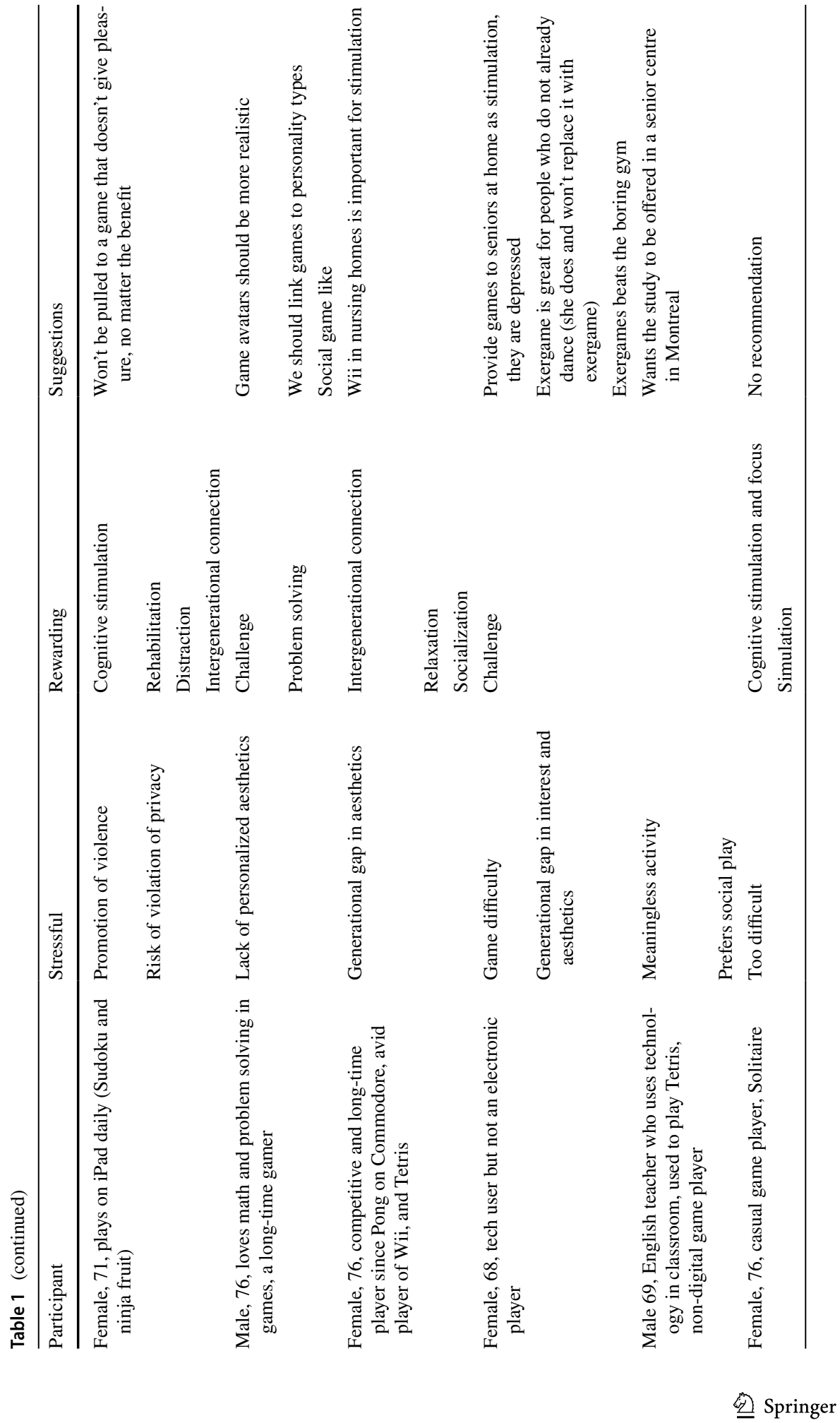




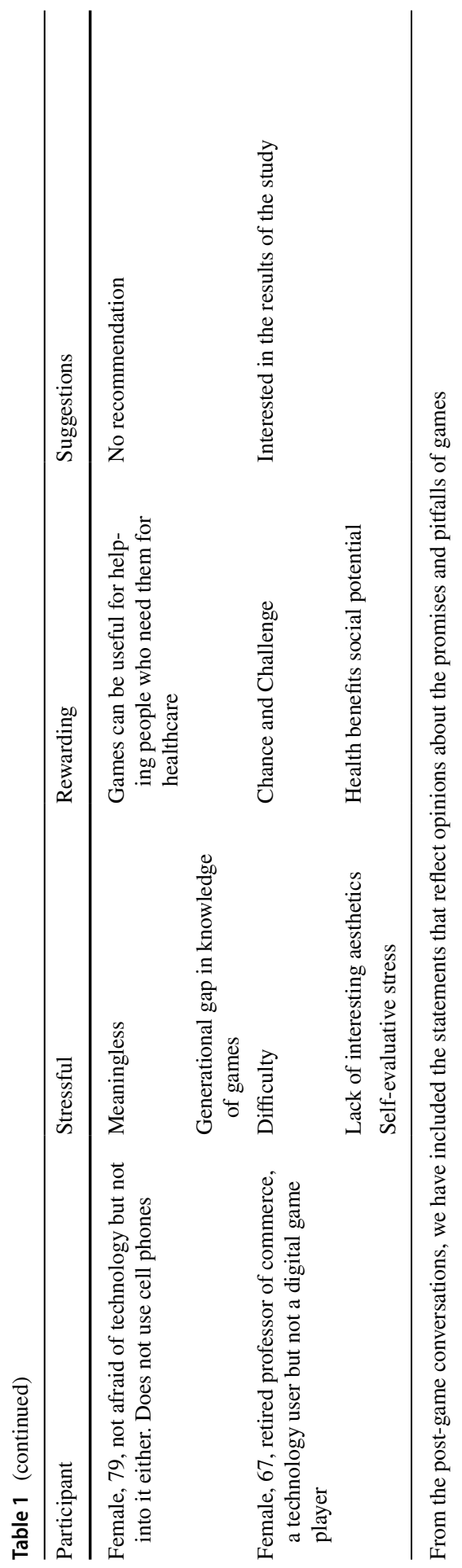


Table 1 summarizes some of these findings. The most salient problems stated were risk of addiction, steep learning curve, generational gap in interest and technology literacy, and lack of appropriate or personalized aesthetics. The most frequently stated rewarding aspects were relaxation, distraction, stimulation and potential for intergenerational connection. A recurring suggestion was that game activities would be useful in senior homes for people who were frailer and socially isolated. Another recurring suggestion was that the interfaces, technical jargon and game instructions needed to be simplified. One participant suggested that this could be remediated by introducing games in libraries.

\subsection{Phase 2: What is Serious About Different Genres of Games?}

Based on the observation in Phase 1 that the value of games for the majority of older adults was limited to their cognitive and relaxing features, and that the generational gap in cultural or aesthetic appreciations of games were pervasive, we conceptualized Phase 2 and set up a community program, a free Game Clinic Course to discuss the potential healthcare applications of serious games. Activities in this course are listed in Table 2. All classes were attended by the PI and at least 3 young students ( $<30$ years of age), and 7 older adult participants ( $>65$ years of age). The same activity was offered in Phase 3 as well, but the emphasis was not just on health. At the end of the class, we asked everyone to write about how this class changed their perspective about games. Specifically, we asked them to reflect on how the class made them connect to their past, and how they envisioned games being relevant to their future. Six of the participants offered short write ups. Excerpts of the writing are presented below:

Table 2 List of different types of games introduced in each class and the context in which they could be used for "serious" outcomes

Meeting 1.: The importance of play

Meeting 2 a: What is serious about games?

Meeting 3: Relaxing games (casual)

Meeting $4^{\text {a }}$ : Virtual civilizations (strategy)

Meeting $5^{\mathrm{a}}$ : Brain training games (attention, reaction, visual processing)

Meeting $6^{\text {a }}$ : Fitness games (exergames)

Meeting 7. : Personality and play (pick your own game)

Meeting 8: Storytelling games (indie games)

Meeting $9^{\text {a }}$ : How they make games?

Meeting 10: Brain computer interface games (presentation by electrical engineering students)

Meeting $11^{\mathrm{b}}$ : Virtual reality

${ }^{a}$ Group 2, in Phase 3 experienced these sessions only

${ }^{\mathrm{b}}$ Only presented in Phase 3 


\subsubsection{Connecting to the Past and Connecting to the Future}

To link the idea of digital games to the experience of play rekindled memories of childhood and provided a new dimension to the ways in which digital games can serve a serious purpose.

Rose (who often talked about her age, 84), an extremely active participant with no history of digital playing prior to joining the class wrote:

When Eileen and Rebecca gave their presentation [about how they programmed games], my brain lit up: [to propose a game about] Storytelling and Scavenger Hunt. [In my childhood] story telling was always about verbal sharing of the stories. My mother played a game where she would shout out: "Once upon a time...", and when we heard that, we would run to her; she would explain the rules, and tell us what we were going to talk about. She would start a story and then pass it on to the next person. We would have to wait until next session to see how we did, but this encouraged us to think as a team about one subject. [Rose, 84 years]

The games that she most enjoyed during our sessions were the car racing, word puzzles and trivia games. She found exercise games to be potentially useful, but not to her, since she was already active in the PERFORM Centre's gym. Additionally, she proposed that a Scavenger Hunt game and an Escape Room would be interesting to her.

Thea (did not reveal her age, but was retired), who preferred the materiality of creativity over any electronic or digital experience, wrote of her childhood being defined by her playful spirit, which saved her in older age from the trauma of sexual assault. About the Game Clinic class, she wrote:

This course has been a godsent to me. (1) It has allowed me to nurture the playful side of me. (2) It has opened me to better understanding and becoming less intimidated by digital technology. (3) It introduced me to a nice group of people-breaking my isolation (in which I am a little too comfortable); giving me something to look forward to; a stimulant for my curiosity and addiction to learning; You are never too old to play or to have fun. [Thea]

Suzan (69, who was also a participant in Phase 1) wrote:

I have to admit that I was surprised that the study (Phase 1) would kindle what is now a renewed interest in gaming. [Suzan, 69 years]

Recalling playing her first internet game in the early 90 s, on their first family computer, Suzan continued:

I didn't have any interest in Pacman, a bunch of creatures eating up blobs, but we had a really interesting game called Where in the World is Carmen Sandiego. This game tests the player's knowledge about different countries and gives clues to the player to figure out where the villain (Carmen Sandiego) has gone in order to arrest her and her allies. [Suzan, 69 years] 
Suzan found the class as a worthwhile activity as it introduced her to several genres of new games that would help her perfect her problem-solving skills, and she was especially intrigued by storytelling games. "It has brought up questions for me about the impact of games on seniors, both good and not so good". She wrote:

Good in that it keeps the mind alert and up to date with the world of the younger generations. Not so good could include addiction to both games and the computer, which could lead to cutting the senior off from the real world. [Suzan, 69 years]

Anna ( $>65$ years of age but did not specify), who described her childhood as a sober one preferentially spent on reading books, reminisced about discovering Wii games with her children and grandchildren. She wrote:

Retirement comes with an endless amount of leisure time. Having discovered the world of games recently has given me new ways to enjoy that time and even to work on mental health issues like memory and concentration. I have new things to talk about with children and grandchildren and have earned some 'creds' for trying out new experiences. Coming to the workshops and sharing play a has changed the way I look at games, not just as something for other people but something I can share with peers. I like the challenge that word or trivia games present, and I like the fun-rush of the racing, or dance games. I'm still trying out the narrative games - I have something called Murder in the Alps on my tablet, but they take a bit more concentration than I have just now. [Anna, $>65$ years]

George, 92, who in the first meeting stated his dislike for computers and technology, acquired his own tablet by the end of the class and downloaded his favorite games (trivia and word puzzles). He wrote:

Since enrolling in (10-weeks) Games, as I am good in spelling, I have found Wordtrip, Wordscapes, Word Search, Word Swipe, Word Stacks. Of course, all these games are challenging and addictive. If one is not careful, these games can cost one a lot of money. To avoid spending money, you can earn rewards by spinning the wheel or watching videos for $30 \mathrm{~s}$. When watching videos, you should divert your eyes off the screen. I will continue to find other games which spike my interest. [George, 92 years]

In fact, this introductory class triggered an interest to offer games as part of a wellness routine in senior's lives. Suzan wrote:

Your idea to open up a gaming center for seniors is fantastic! [...] to build some community events into this center which could include teaching people about new games, how to play them, etc. Sort of like an ongoing game clinic! [It] could help out less fortunate seniors who can't afford a computer or tablet. Often school boards change their computers when the existing ones are still functional. Maybe a donation system could be set up. [Suzan, 69 years] 


\subsection{Phase 3: What Older Adults Taught our Design Students?}

For the third and final phase, we repeated the same process as described for Phase 2 , but invited $65+$ year old individuals to participate in a research project, to help us find what is the serious in which type of game. In this phase, we explicitly shifted away the discussion about games from the health context and towards critical reflections about the significance of the games as cultural and communicative media. We were interested in identifying first, what constitutes the "serious" in the playing, and also what may be the cultural barriers to adoption of digital games in the lifestyles of older adults.

\subsubsection{Who Were the Study Participants and What They Wanted?}

We ran two sets of classes for Group 1, and Group 2. Those who joined our third call were younger, recently retired, and proficient with usage of technology. The responders were mostly women. Group (1) included a retired journalist, a retired computer programmer, a retired literature teacher (male), a working graphic designer, and two young game studies and one young engineering graduate students (Age $<30$ years).

Group (2) included a retired designer, a retired social worker, a retired professor, a retired journalist and working writer, a self-described hermit (sex male, gender non-binary), and a working yoga teacher (age range 65-75), and four students from creative art therapy (age range 30's), none of whom were familiar with or previously interested in games.

Our first question to the participants was "What interested you to join the study?" The majority indicated their interest in 'finding a cognitively stimulating activity', 'curiosity about the topic', and 'desire to remain engaged in what the young research these days.'

\subsubsection{Cultural Stereotypes Exclude Seniors from Game Culture}

The first important finding of this phase was that that the gamer culture is not inclusive to older adults (nor to younger adults who were not interested in games and had a negative opinion of games to begin with). One of our young game designers (student in Communication Studies) remarked:

My experiences as a girl who plays video games cross over with the experiences of these seniors in many ways. Video games tend to be marketed to young masculine audiences (Carly Kocurek 2015). Although this is being challenged by indie games and casual games, the spaces for different kinds of play in video games are often not communicated to these audiences. After all the sessions together, most of our participants admitted to being interested in playing more video games, but not knowing how to access them. Part of the challenge here, and that we realized throughout the workshop, is that there are not many efforts made to advertise to older audiences. 
Another game designer (student in Computation Arts), who was introduced to gaming by her grandmother "wielding magic and fighting evil in games like The Legend of Zelda", commented that many negative stereotypes around gender and age permeated the medium of game. She admitted that the experience in the game clinic revealed that she too "was not immune to presuming that she knew her [older] gaming audience". She was surprised to learn how niche game design marketing remains:

Although the participants greatly enjoyed trying the games that we shared, they had mostly not heard of them at all prior to this intervention. What this means to me as a game designer is that many groups of people, particularly our seniors, continue to be alienated from our play experiences that are mostly marketed online.

\subsubsection{Cultural Accessibility is More Important Than Mechanical Accessibility}

As expected, it was clear that the younger game players underestimated the difficulty of game mechanics for older players. One (Communication Studies) wrote:

The more accessible games were always easier for participants to get invested in than those that required knowing how to use a controller or a keyboardknowledge I realized I take for granted as someone who grew up with these technologies.

Another game designer student commented on a need to simplify instructions:

When designing games, we the designers often assume that certain conventions are known by the prospective player; that ' $A$ ' means jump, that you move left to right across the screen, etc. However, for people who have little to no prior experience with games, these things are not known. Moreover, assuming and failing to explain these conventions alienates new players from being able to fully enjoy an experience.

A third student (Communication Studies) noted that to presume that technology is the only impediment to game adoption by older adults, blinds us to the fact that we do not take time to reach out and teach them about the affordances of games:

While many of the seniors that came to the program were relatively tech savvy, they showed interest in what new technologies were being developed and wanted to understand the appreciation that others had towards tech. To clarify, they were not inept at technology, but were curious to other interactions they could have with it.

One of the most important findings in Phase 3 was for students to discover that access is too often overlooked not only in game design, but also in game marketing and dissemination. For instance, our participants were not familiar with indie games. When we introduced games such as the Old Man's Journey, Lieve Oma, or Night in the Woods, they found them as interesting story-telling techniques, even though they lacked in the polished visual pleasures of a video-based story, and the excitement 
and short-term winnable excitements of games. Nevertheless, they acknowledged the artistic and creative potential of the indie games.

That games could be personalized was the feature that triggered most imaginations. One participant in Phase 1, a highly active one, still working as a freelance accountant, and a competitive (social) dancer of 79 years of age, emphasized that although she recognized the potential value of games for rehabilitation and cognitive training, she would still not play games for fun (unless they became personalized-for instance if the music and the genre of dance game were selected from her youth). The most poignant point made by another participant was:

if you want to make a game for seniors, make one for my 90 -year-old mother, that makes her believe she is improving her scores, even if she is not scoring higher.

The idea that games had the possibility of being customized to not only physical needs, but also cultural preferences of users was intriguing to participants. One participant (the male who called himself a hermit), was very familiar with Huizinga and emphasized the distinction between play and games. He was completely uninterested in games (and in fact refused to play any). Yet, he indicated that he took part in our class because he believed that the youth were spending too much time on computers, and he wanted to encourage game makers to create games that promote cooperation, towards solving the climate crisis. Thus, the intergenerational gaps were not only in mechanical accessibility of the game, but also in stereotypical misunderstandings and cultural disconnections about the definition of games.

\subsubsection{Creating Safe Spaces to Learn and Trust is Important}

In Phase 3, two participants dropped out after the first session: one, because our activity was not geared towards brain health; second, because the individual preferred to exercise. Both these individuals had come to the class with a negative attitude towards games, as being wasteful, and mind-numbing. However, the ten who remained (including the 'hermit' who came in with a critically constructive attitude), demonstrated remarkable willingness and flexibility to revisit some of their beliefs about the addictiveness and uselessness of certain games. Some of the participants were shyer than others in testing out new technologies, but everyone tried them-unless they perceived a physical risk of falling, for example in playing the exergames.

A student commented:

I think part of the reason behind their willingness was that the Game Clinic provided a very safe space to mess around with and learn different technologies, without judgment. But this work did not stop at the workshop level; participants also admitted to downloading some of the games at home and continuing to play on their own time. They also spoke about having the ability to now talk to their grandchildren about their games and finding value in these conversations. The workshop even inspired some of the participants to get 
more involved in creative and academic practices on their own time, such as writing stories, creating worlds, as well as signing up for further classes.

This was reiterated by another student:

Watching participants consistently return, learn, experience, and share their thoughts on games created a meaningful space for dialogue. By creating a space welcoming to new game players, the clinic allowed older adults to bring in their ideas and opinions into the space. As a facilitator, watching people interacting with games, helping them understanding the games expectations and supporting them in their play (as ally or rival), created a positive learning and knowledge dissemination environment for the study.

This observation by students was validated by an E-lecture (via skype at the Milieux Institute for Arts, Culture and Technology) conducted by Bob de Schutter. He presented the topic of Gaminiscing (reminiscing via a game), and gave a demonstration of the game play, but also the process of creating his new game: Brukel - a game he created based on his grandmother's reminiscing about her childhood in Belgium, in the frontlines of the Second World War, when her family's house had come under the Nazi Occupation (https://www.brukelgame.com/.) This presentation was lauded as the highlight of all other activities-until we presented the VR in the next session. When asked about the conclusion of this class, one participant said: "I had no clue that games are so varied, and because they are so varied, it is hard to give only one impression about them."

Another participant wished she had brought her game-playing grandchildren along (they were at all times invited) to this session. Discussions after this phase continued well after the class had adjourned-and everyone was proposing either a narrative for a similar game from their personal experience, or alterations to the Brukel: "It would be interesting if the game also included a few scenes from the perspective of the German soldier", one said.

\subsection{What Kind of Serious Games Would Matter?}

In the final session of the class we offered a virtual reality (VR) tour guided by a VR artist and educator at the Milieux Institute for Art, Culture and Technology (Marco Antonio Luna Brahona). We presented a range of VR experiences in the following order (theBlu by Wevr Inc.; Pearl by Google Inc., the Lab by Valve, and Tilt Brush by Google). While these experiences were not games (other than the Lab), they provided an opportunity for presenting even more technologically complex media experiences that could be gamified and invited all to imagine such possibilities.

Indeed, participants immediately drew a connection between VR and Brukel, as one indicated: "This technology might let you see the perspective of others in the last week's game". Three participants found the experience of theBlu of therapeutic value. Two participants who tried Pearl talked about how they felt emotional about the story. After Pearl, one commended that the immersive nature of the medium might have a potential for curing racism. She also discussed how she 
wished someone reconstructed into VR, a heritage building (now turned into luxury condominiums) in which she worked as a young girl. This idea was reiterated by another participant who offered a history tour of Montreal (in Group 1 of Phase 3). He thought that VR had a great potential for teaching history, or providing people access to museums and galleries that they could not travel to.

At the end, Noah (70 years) who participated in Phase 1 and the three last sessions of Phase 3 (a social player, but not a digital player) reiterated his commitment to help bring our class to a local senior's community centre. Two participants from the second group of Phase 3, indicated that they wanted to write to the university to ask for the class to be extended. When we mentioned that classes were going to happen in a living lab (that will be set up in a mall with high number of senior customers), two offered to tend to the space as volunteers and guides. The prospect of having access to the VR in that space was most appealing-reiterating the point that to provide access and education about new technologies should be prioritized for seniors.

\section{Discussion}

The aim of this study was to gain an understanding of how older adults relate to the notion of digital serious games as innovative technologies for enhancing their health and wellbeing. We first framed the study in the context of a neuroscientific and biomedical engineering inquiry and invited older adults to help us "find better games" for their age group. The results of our first study led us to acknowledge a need for addressing a cultural gap. By creating a safe space for teaching about and criticizing digital games, we explored the familiarity, appeals or barriers for adoption of digital games into the lifestyle of older adults in the next phases of the study.

Comments, conversations and written statements generated by both older players, and younger game-makers, collected over more than $100 \mathrm{~h}$ of intergenerational discussions point out to the following main findings:

1. Initially, cognitive stimulation, relaxation, and physical exercise were the most commonly stated justification for digital game playing by seniors.

2. The more we conversed about games, the more clear it became that in addition to physical (mechanical) accessibility, intergenerational barriers stemming from cultural inaccessibility of games need to be addressed.

3. In addressing the question of "What is serious about digital games?", the frequently stated possibilities included: problem solving, storytelling, learning and skill development, and importantly, history teaching and creating invigorating social interactions around playing with an interactive and immersive technology (e.g. Nintendo Wii console or VR). 


\subsection{Significance of the Study}

The significance of this work is that we created a safe space for exploration of several genres of commercially available games, without burdening our participants with any request to engage in any activity outside their comfort. We did not state any implicit expectation about the benefits of any particular genre of games, nor measured performance. Instead, by encouraging our older participants to be critical about what does and what does not work in various types of games, we were able to uncover an important issue which is often neglected in health-game studies: The cultural valence of games. Importantly, we found that, when games were presented in a cultural context (as an artistic medium) the interest in games transcended the cliché expectations of cognitive stimulation, distraction and physical therapy.

Our work provides additional support for the importance of an intergenerational approach to game research by tackling the issue from the perspectives of increasing inclusion and diversity, through the processes of co-design (Vanden Abeele and De Schutter 2010; Kaufman et al. 2017; Derboven et al. 2011), situated learning and increasing social interaction by co-playing among different age groups (Zhang et al. 2017; Chua et al. 2013). For review of previous work, see (Costa and Veloso 2016; Zhang and Kaufman 2016b; de la Hera et al. 2017).

In this study, instead of focusing on a single game, or a genre of games, we provided a space for exploring different possibilities of using games as modes of communication and invited older participants to critique whether games could be pertinent to their lifestyle. This approach is different from previous multi-session studies like Zhang et al. (2017), in that we used the context of healthcare applications of serious games as a point of departure to understand the cultural relevance of serious games in a safe space without expectations to learn, perform or adopt any one particular game.

Our observations reiterate what has long been known among gerontoludic designers: the need for accessible designs (Gerling et al. 2011; Bouchard et al. 2012), games which are mentally challenging (Cota et al. 2015), satisfy needs for self-efficacy and connection (De Schutter 2017), and are offered by continuous interactive engagement (Hausknecht et al. 2015).

Additionally, we found that the main impediment to the adoption of games by older adults is a lack of understanding of the cultures that permeate the medium of game. When given the option of picking a hobby or health-improving activity, the majority of seniors did not pick games. However, digital games intrigued them for their potential to help intergenerational connections. Such possibilities are yet to be explored.

Contrary to our assumptions, it was not so much the idea of gaming for health that attracted those who participated in this work. Similar to previous findings, actual physical activity was stated to be more important than any game for healthy lifestyle (Liechty et al. 2016). Even though our participants assumed that games could be good for mental stimulation, it was their desire to learn, and to keep in touch with contemporary research on games that compelled them to help our study. To bridge between generations was frequently mentioned in reference to playing with grand-children (for those who had them), and for keeping in conversation with the 
young (in those who joined the class to tell the young to move away from combative shooter games, and design socially responsible games.)

The success of our Game Clinic Class has provided us the opportunity to seek support for transferring these activities to a shopping mall (https://www.concordia. $\mathrm{ca} /$ news/stories/2020/01/13/concordias-engage-centre-launches-the-creative-livin g-lab-at-a-local-storefront.html), where we will have an opportunity to further test the conclusions we have reached so far.

\subsection{Limitations}

Our study has a major limitation that future work can address. The study recruited participants through PERFORM Centre's mailing lists, whose subscribers are interested in preventative health and wellness studies. As such, our sample consisted of generally high socioeconomic status, who are well-educated people with an age range skewed towards younger seniors-a problem that permeates studies of this kind (Marston et al. 2016). Our participants (with the exception of two) were all Caucasian, and in good physical and mental health. We plan to address these limitations by transferring our class to a living lab in a shopping mall with a high population of seniors as the customer base. Whether our study will attract participants from minority communities and from older adults, or from younger students who will have to conduct the classes outside the university campus, remains to be seen.

\subsection{Recommendations}

Both our older participants and younger students concurred that currently, there are no spaces for engaging in intergenerational exchange about games.

In our observations, the largest gap between generations seem to be caused by mutual misconceptions and stereotypical pre-judgements about games. Many in the older generations generally view games as addictive and violent, with the only positive potential for cognitive training, thanks to broad marketing of Lumosity (which was frequently referred to by several of our study participants as a selfcare activity) despite dispute about its cognitive benefits (Simons et al. 2016). Many in the younger generation also neglect that to play is equally important to older adults, and that accessibility in games needs to also consider marketing strategies and content creation to connect the similarities of experiences lived across a lifespan.

We recommend that this gap should be bridged by:

- Creating academic curricula that mandate intergenerational co-learning and cocreation projects in digital design.

- Funding community programs that provide seniors training for, and access to, both hardware and software used by the latest information and communication technologies.

- Prioritizing research and development of games that address the needs of seniors for intergenerational, and intercultural communication and socialization. 


\section{Conclusion and Future Work}

We used the approach in Jaycox and Hicks' (1976) study (Jaycox and Hicks 1976), which aimed to bridge an intergenerational gap by sensitizing the young students in developing and using 'humanized' computer programs. One of the findings of their study was that older adults' imagination of the utility of computers transcended the applications they were intended for, as they found the technology useful to share experience and knowledge with younger generations by using hypertexts and simulated environments.

Similarly, we learned that the expectations of older adults from digital games can go beyond their often advertised mental and physical health benefits. When minor steps were taken to have an openminded conversation about games, then seniors found them most serious, as tools to communicate with the young, to re-construct the past, and to live unattainable experiences (such as immersing in the ocean via virtual reality, and skiing via Wii technologies.)

We conclude that intergenerational cultural gaps are a greater impediment to technology adoption than accessibility alone and recommend that to create an environment in which different age groups can exchange ideas about games should be an educational priority.

Acknowledgements The authors wish to thank Kate Li and Alessia Signorino for transcribing discussions in Phases 1 and 3, respectively; Dr. Sujaya Dhanvantari for research and feedback on Phase 3 (in preparation for the Mall project), and for proof-reading of the manuscript; and Ms Gina Haraszti for logistic support. Financial support for this work is provided by a seed grant from the Office or Research (Concordia University), and Webster Foundation, as well as by equipment support from the Milieux Institute for Art, Culture and Technology (Technoculture, Arts and Games cluster). Phase 3 of this study was partially supported by FRQ-S (engAGE Living Lab: Meet me at the Mall).

Authors' Contributions NK-M prepared the study protocols, obtained ethics approval, conducted the interviews, and wrote the manuscript. BdS \& KM co-wrote the manuscript. MM, EMH, RG, SdJ taught classes in Phase $2 \& 3$, took memos, and contributed to writing. SM and RM were participant advisors on the study.

\section{Compliance with Ethical Standards}

Conflict of interest The authors do not have any conflict of interest to disclose.

Open Access This article is licensed under a Creative Commons Attribution 4.0 International License, which permits use, sharing, adaptation, distribution and reproduction in any medium or format, as long as you give appropriate credit to the original author(s) and the source, provide a link to the Creative Commons licence, and indicate if changes were made. The images or other third party material in this article are included in the article's Creative Commons licence, unless indicated otherwise in a credit line to the material. If material is not included in the article's Creative Commons licence and your intended use is not permitted by statutory regulation or exceeds the permitted use, you will need to obtain permission directly from the copyright holder. To view a copy of this licence, visit http://creativecommons.org/licen ses/by/4.0/. 


\section{References}

Allaire, J. C., McLaughlin, A. C., Trujillo, A., Whitlock, L. A., Laporte, L., \& Gandy, M. (2013). Successful aging through digital games: Socioemotional differences between older adult gamers and non-gamers. Computers in Human Behavior, 29, 1302-1306.

Basak, C., Boot, W. R., Voss, M. W., \& Kramer, A. F. (2008). Can training in a real-time strategy video game attenuate cognitive decline in older adults? Psychology and Aging, 23, 765-777.

Bleakley, C. M., Charles, D., Porter-Armstrong, A., McNeill, M. D., McDonough, S. M., \& McCormack, B. (2015). Gaming for health: A systematic review of the physical and cognitive effects of interactive computer games in older adults. Journal of Applied Gerontology, 34, NP166-89.

Boot, W. R., Souders, D., Charness, N., Blocker, K., Roque, N. \& Vitale, T. (2016). The gamification of cognitive training: Older adults' perceptions of and attitudes toward digital game-based interventions. In Human aspects of it for the aged population: Design for aging, Itap 2016, Pt I (Vol. 9754, pp. 290-300).

Bouchard, B., Imbeault, F., Bouzouane, A., \& Menelas, B.-A. J. (2012). Developing serious games specifically adapted to people suffering from Alzheimer (pp. 243-254). Berlin: Springer.

Chesham, A., Wyss, P., Müri, R. M., Mosimann, U. P., \& Nef, T. (2017). What older people like to play: Genre preferences and acceptance of casual games. JMIR Serious Games, 5, e8.

Chua, P.-H., Jung, Y., Lwin, M. O., \& Theng, Y.-L. (2013). Let's play together: Effects of video-game play on intergenerational perceptions among youth and elderly participants. Computers in Human Behavior, 29, 2303-2311.

Costa, L., \& Veloso, A. (2016). Being (grand) players: Review of digital games and their potential to enhance intergenerational interactions. Journal of Intergenerational Relationships, 14, 43-59.

Cota, T. T., Ishitani, L., \& Vieira, N. (2015). Mobile game design for the elderly: A study with focus on the motivation to play. Computers in Human Behavior, 51, 96-105.

Coughlan, G., Coutrot, A., Khondoker, M., Minihane, A. M., Spiers, H., \& Hornberger, M. (2019). Toward personalized cognitive diagnostics of at-genetic-risk Alzheimer's disease. Proceedings of the National Academy of Sciences United States of America, 116, 9285-9292.

de la Hera, T., Loos, E., Simons, M., \& Blom, J. (2017). Benefits and factors influencing the design of intergenerational digital games: A systematic literature review. Societies, 7, 18.

de Schutter, B. (2011). Never too old to play: The appeal of digital games to an older audience. Games and Culture, 6, 155-170.

de Schutter, B. (2017). Gerontoludic design: Extending the MDA framework to facilitate meaningful play for older adults. International Journal of Gaming and Computer-Mediated Simulations, 9, 45-60.

de Schutter, B., \& vanden Abeele, V. (2015). Towards a gerontoludic manifesto. Anthropology \& Aging, 36, 112-120.

de Vette, F., Tabak, M., Dekker-van Weering, M., \& Vollenbroek-Hutten, M. (2015). Engaging elderly people in telemedicine through gamification. JMIR Serious Games, 3, e9.

Derboven, J., van Gils, M., \& de Grooff, D. (2011). Designing for collaboration: A study in intergenerational social game design. Universal Access in the Information Society, 11, 57-65.

Deterding, S., Dixon, D., Khaled, R. \& Nacke, L. (2011). From game design elements to gamefulness. In Proceedings of the 15th international academic MindTrek conference on envisioning future media environments-MindTrek' 11 .

Gerling, K. M., Mandryk, R. L. \& Linehan, C. (2015). Long-term use of motion-based video games in care home settings. In Chi 2015: Proceedings of the 33rd annual Chi conference on human factors in computing systems (pp. 1573-1582).

Gerling, K. M., Schulte, F. P. \& Masuch, M. (2011). Designing and evaluating digital games for frail elderly persons. In Proceedings of the 8th international conference on advances in computer entertainment technology (Ace 2011).

Hall, A. K., Chavarria, E., Maneeratana, V., Chaney, B. H., \& Bernhardt, J. M. (2012). Health benefits of digital videogames for older adults: A systematic review of the literature. Games Health J, 1 , $402-410$.

Hausknecht, S., Schell, R., Zhang, F. \& Kaufman, D. (2015). Older adults digital gameplay: A followup study of social benefits. In Information and communication technologies for ageing well and e-health, Ict4ageingwell 2015 (Vol. 578, pp. 198-216).

Ijsselsteijn, W., Nap, H. H., Kort, Y. D. \& Poels, K. (2007). Digital game design for elderly users. In Proceedings of the 2007 conference on Future Play. Toronto: ACM. 
Jaycox, K. \& Hicks, B. (1976). Elders, students \& computers background information [Online]. Washington, DC: ERIC Clearinghouse. Retrieved January 1, 2020, from https://eric.ed.gov/?id=ED138285.

Kaufman, D., Owens, H., Jeremic, J., Zhang, F. \& Hausknecht, S. (2017). Digging into game design for older adults-Collaborative user-centered game design with postsecondary students. In Proceedings of the 9th international conference on computer supported education.

Kaufman, D., Sauve, L., Renaud, L., Sixsmith, A., \& Mortenson, B. (2016). Older adults' digital gameplay: Patterns, benefits, and challenges. Simulation \& Gaming, 47, 465-489.

Khalili-Mahani, N., Assadi, A., Li, K., Mirgholami, M., Rivard, M.-E., Benali, H., et al. (2020). A mixed-methods study of the reflective and reflexive stress responses of older Adults to three gaming experiences in relation to their cognitive abilities. JMIR Mental Health, 7(3), e12388. https://doi. org/10.2196/12388.

Khalili-Mahani, N., \& de Schutter, B. (2019). Affective game planning for health applications: Quantitative extension of gerontoludic design based on the appraisal theory of stress and coping. JMIR Serious Games, 7, e13303.

Lee, H., Voss, M. W., Prakash, R. S., Boot, W. R., Vo, L. T., Basak, C., et al. (2012). Videogame training strategy-induced change in brain function during a complex visuomotor task. Behavioural Brain Research, 232, 348-357.

Liechty, T., Genoe, M. R., \& Marston, H. R. (2016). Physically active leisure and the transition to retirement: The value of context. Annals of Leisure Research, 20, 23-38.

Loos, E., \& Kaufman, D. (2018). Positive impact of exergaming on older adults' mental and social wellbeing: In search of evidence (pp. 101-112). Cham: Springer.

Marston, H. R., Freeman, S., Bishop, K. A., \& Beech, C. L. (2016). A scoping review of digital gaming research involving older adults aged 85 and older. Games for Health Journal, 5, 157-174.

Marston, H. R. \& Hall, A. K. (2016). Gamification. In Handbook of research on holistic perspectives in gamification for clinical practice.

Marston, H. R., Kroll, M., Fink, D., \& Gschwind, Y. J. (2015a). Flow experience of older adults using the iStoppFalls exergame. Games and Culture, 11, 201-222.

Marston, H. R., \& Smith, S. T. (2012). Interactive videogame technologies to support independence in the elderly: A narrative review. Games for Health Journal, 1, 139-152.

Marston, H. R., Woodbury, A., Gschwind, Y. J., Kroll, M., Fink, D., Eichberg, S., et al. (2015b). The design of a purpose-built exergame for fall prediction and prevention for older people. European Review of Aging and Physical Activity, 12, 13.

Mcguire, F. A. (2010). Improving the quality of life for residents of long term care facilities through video games. Activities, Adaptation \& Aging, 6, 1-7.

Miller, K. J., Adair, B. S., Pearce, A. J., Said, C. M., Ozanne, E., \& Morris, M. M. (2014). Effectiveness and feasibility of virtual reality and gaming system use at home by older adults for enabling physical activity to improve health-related domains: a systematic review. Age and Ageing, 43, 188-195.

Ouellet, É., Boller, B., Corriveau-Lecavalier, N., Cloutier, S., \& Belleville, S. (2018). The Virtual Shop: A new immersive virtual reality environment and scenario for the assessment of everyday memory. Journal of Neuroscience Methods, 303, 126-135.

Riddick, C. C., Drogin, E. B., \& Spector, S. G. (1987). The impact of videogame play on the emotional states of senior center participants. The Gerontologist, 27, 425-427.

Simons, D. J., Boot, W. R., Charness, N., Gathercole, S. E., Chabris, C. F., Hambrick, D. Z., et al. (2016). Do "brain-training" programs work? Psychological Science in the Public Interest, 17, 103-186.

Vallejo, V., Wyss, P., Chesham, A., Mitache, A. V., Müri, R. M., Mosimann, U. P., et al. (2017). Evaluation of a new serious game based multitasking assessment tool for cognition and activities of daily living: Comparison with a real cooking task. Computers in Human Behavior, 70, 500-506.

vanden Abeele, V., \& de Schutter, B. (2010). Designing intergenerational play via enactive interaction, competition and acceleration. Personal and Ubiquitous Computing, 14, 425-433.

Weisman, S. (1983). Computer games for the frail elderly. Gerontologist, 23, 361-363.

Whitcomb, G. R. (1990). Computer games for the elderly. SIGCAS Computers and Society, 20, 112-115.

Whitlock, L. A., Patterson, T., Trujillo, A., McLaughlin, A., Gandy, M., \& Allaire, J. C. (2011). Older adults' perception of the benefits associated with intervention-based video game play. Gerontologist, 51, 40 .

Zhang, F., \& Kaufman, D. (2016a). Physical and cognitive impacts of digital games on older adults: A meta-analytic review. Journal of Applied Gerontology, 35, 1189-1210.

Zhang, F., \& Kaufman, D. (2016b). A review of intergenerational play for facilitating interactions and learning. Gerontechnology, 14, 127-138. 
Zhang, F., Kaufman, D., Schell, R., Salgado, G., Seah, E. T. W., \& Jeremic, J. (2017). Situated learning through intergenerational play between older adults and undergraduates. International Journal of Educational Technology in Higher Education, 14, 1-6.

\section{Affiliations}

Najmeh Khalili-Mahani $i^{1,2,3,4,7}$. Bob De Schutter ${ }^{5}$ Mahsa Mirgholami ${ }^{1,4}$. Eileen Mary Holowka, ${ }^{3,6}$. Rebecca Goodine ${ }^{3}$. Scott DeJong ${ }^{3,6}$. Roseleen McGaw ${ }^{8}$. Sue Meyer ${ }^{8} \cdot \mathrm{Kim}$ Sawchuk ${ }^{2,3,6}$

1 PERFORM Centre, Concordia University, Montreal, QC, Canada

2 engAGE Centre for Studies in Aging, Concordia University, Montreal, QC, Canada

3 Technoculture, Art \& Games Cluster (TAG), Milieux Institute for Art, Culture and Technology, Concordia University, Montreal, QC, Canada

4 Department of Electrical and Computer Engineering, Concordia University, Montreal, QC, Canada

5 Armstrong Institute for Interactive Media Studies, Miami University, Oxford, OH, USA

6 Department of Communication Studies, Concordia University, Montreal, QC, Canada

7 Department of Design and Computation Arts, Concordia University, Montreal, QC, Canada

8 Montreal, QC, Canada 